Investigation of the effects of transcranial alternating current stimulation (tACS) on self-paced rhythmic movements

Manuel Varlet ${ }^{1, *}$, Alanna Wade ${ }^{1}$, Giacomo Novembre $^{1,2}$, \& Peter E. Keller ${ }^{1}$

${ }^{1}$ The MARCS Institute for Brain, Behaviour and Development, Western Sydney University, Australia

${ }^{2}$ Department of Neuroscience, Physiology, and Pharmacology, University College London, United Kingdom

* Corresponding author. Address: The MARCS Institute for Brain, Behaviour and Development, Western Sydney University, Locked Bag 1797, Penrith NSW 2751, AUSTRALIA

E-mail address: $\underline{\text { M.Varlet@ westernsydney.edu.au }}$ 


\begin{abstract}
Human rhythmic movements spontaneously entrain to external rhythmic stimuli. Such sensory-motor entrainment can attract movements to different tempi and enhance their efficiency, with potential clinical applications for motor rehabilitation. Here we investigate whether entrainment of self-paced rhythmic movements can be induced via transcranial alternating current stimulation (tACS), which uses alternating currents to entrain spontaneous brain oscillations at specific frequencies. Participants swung a handheld pendulum at their preferred tempo with the right hand while tACS was applied over their left or right primary motor cortex at frequencies equal to their preferred tempo (Experiment 1) or in the alpha $(10 \mathrm{~Hz})$ and beta $(20 \mathrm{~Hz})$ ranges (Experiment 2$)$. Given that entrainment generally occurs only if the frequency difference between two rhythms is small, stimulations were delivered at frequencies equal to participants' preferred movement tempo $(\approx 1 \mathrm{~Hz})$ and $\pm 12.5 \%$ in Experiment 1, and at $10 \mathrm{~Hz}$ and $20 \mathrm{~Hz}$, and $\pm 12.5 \%$ in Experiment 2. The comparison of participants' movement frequency, amplitude, variability, and phase synchrony with and without tACS failed to reveal entrainment or movement modifications across the two experiments. However, significant differences in stimulation-related side effects reported by participants were found between the two experiments, with phosphenes and burning sensations principally occurring in Experiment 2, and metallic tastes reported marginally more often in Experiment 1. Although other stimulation protocols may be effective, our results suggest that rhythmic movements such as pendulum swinging or locomotion that are low in goal-directedness and/or strongly driven by peripheral and mechanical constraints may not be susceptible to modulation by tACS.
\end{abstract}

Keywords: tACS; entrainment; self-paced rhythmic movement; frequency-dependent side effects. 
Like most biological systems, human movement systems are characterized by rhythmic or periodic activity (von Holst, 1973; Kugler \& Turvey, 1987; Kelso, 1995). The rhythmicity of human movements is clear when observing someone walking, running, cycling or performing more complex activities such as dancing or playing music, for example. Depending on personal biomechanical and neurophysiological properties, individuals have a preferred tempo or frequency when producing such rhythmic movements (von Holst, 1973; Kugler \& Turvey, 1987; Beek et al., 1995; Nessler et al., 2009). Although this preferred tempo is quite stable over time, a particularly interesting property of human rhythmic movements, and biological rhythms more generally, is that they tend to entrain spontaneously to other rhythms (von Holst, 1973; Pikovsky et al., 2003; Schmidt \& Richardson, 2008; Nessler et al., 2009).

Previous research has shown that rhythmic movements entrain to auditory and visual rhythms in the environment and that such sensorimotor entrainment can be used to improve motor efficiency by attracting movements to different tempi and increasing movement stability in time and space. These findings open promising avenues for using entrainment to improve motor performance, and locomotion patterns in particular, of post-stroke or Parkinson's patients (Thaut et al., 1996; McIntosch et al., 1997; Nombela et al., 2013; Hove $\&$ Keller, 2015). In the current study we investigate in two experiments whether transcranial alternating current stimulation (tACS) can be used to entrain and modulate the dynamics of spontaneous rhythmic movements. tACS is a noninvasive method that allows neuronal excitability and spontaneous brain oscillations to be modulated via low intensity alternating (rhythmic) currents applied on the scalp surface (Joundi et al., 2012; Antal \& Paulus, 2013; Reato et al., 2013; Herrmann, et al., 2015; Helfrich et al., 2015). Here we aim to determine whether entrainment that typically occurs with auditory and visual sensory rhythms can be induced by this method instead.

Entrainment of human rhythmic movements has been demonstrated in a wide variety of situations. Entrainment occurs intrapersonally when an individual performs multiple motor rhythms simultaneously, such as in bimanual coordination situations where the movements of the right and left arms of an individual tend to spontaneously synchronize together (Kelso et al., 1979; Kelso, 1984; Kelso, 1995; Schmidt et al., 1998). Entrainment also occurs interpersonally between interacting individuals when talking, walking, running or applauding together, for instance (Schmidt \& O’Brien, 1997; Néda et al., 2000; Richardson et al., 2007; Nessler et al., 2009; Coey et al., 2011; Varlet et al., 2014; Varlet \& Richardson, 2015). In 
these situations, body sway, arm or leg movements of interaction partners become entrained when there is an exchange of visual and/or auditory information. Human rhythmic movements also entrain to non-human external rhythms in the environment such as computergenerated sequences of visual and auditory stimulus (Repp \& Penel, 2004; Schmidt et al., 2007; Varlet et al., 2015, 2016).

Previous research has shown that spontaneous entrainment of human rhythmic movement preferentially occurs when the frequency of the external rhythm is close to the individual's preferred movement frequency (Schmidt \& O’Brien, 1997; Richardson et al., 2007; Varlet et al, 2015). Entrainment is therefore strongest when differences between an individual's preferred frequency and the frequency of an external rhythm are near zero. Entrainment decreases when the difference between the two frequencies increases, eventually vanishing when the frequency difference is large. By examining the entrainment between the movements of participants swinging a handheld pendulum and an oscillating visual stimulus on a projection screen, previous research has shown that entrainment generally occurs if the difference between the two frequencies does not exceed 15-20\% (Lopresti-Goodman et al., 2008). When the frequency difference between the two rhythms becomes too large to be compensated for by the sensory coupling, they proceed independently of each other at their preferred tempo. Entrainment can then reappear when the frequency difference between the two rhythms reaches stable multi-frequency ratios such as 1:2, 2:3 or 3:4 (Washburn et al., 2014).

Entrainment also underlies the effects of tACS on brain dynamics. Via alternating direct-currents, tACS can entrain brain oscillations and modulate their magnitude, frequency and phase (Feurra et al., 2011; Helfrich et al., 2014; Herrmann et al., 2015). A variety of effects on brain oscillations and the cognitive functions that they underpin have been reported with this method (see Antal and Paulas (2013), Fröhlich (2014), Hermann et al. (2015) for reviews). Effects of tACS on motor functioning have also been demonstrated (Pogosyan et al., 2009; Wach et al., 2013; Pollok et al., 2015). Previous research has shown that the size of the Motor Evoked Potentials (MEPs) induced by Transcranial Magnetic Stimulation (TMS) can be enhanced with tACS when applied over the primary motor cortex (M1) (Antal et al., 2008; Feurra et al., 2011; Keil et al., 2014; Guerra et al., 2016; Nakazono et al., 2016; Raco et al., 2016). Effects of tACS on the speed and accuracy of movements have been reported in a variety of tasks, including fast finger-tapping (Pogosyan et al, 2009; Wach et al., 2013). Previous research has also shown that tACS can modulate tremor magnitude in Parkinson's 
patients (Pogosyan et al., 2010; Brittain et al., 2013; Krause et al., 2013). However, despite the multiple effects of tACS reported on motor processes, it remains largely unknown whether the entrainment and modulation of self-paced rhythmic movements can be induced by tACS, and if so, by which stimulation frequencies.

Brain oscillations underpinning the production and control of self-paced rhythmic movements remain poorly understood given that previous studies using neuroimaging techniques have revealed changes in brain oscillations at multiple frequency levels (Kelso et al., 1998; Cheyne et al., 2008; Bourguignon et al., 2012; Nozaradan et al., 2014).

Electroencephalography and Magnetoencephalography studies have shown enhanced magnitude of brain oscillations in the contralateral primary motor cortex of a moving hand at the frequency of the movement produced $(\approx 1-3 \mathrm{~Hz})$, and with lower magnitudes, at its first harmonics - positive integer multiples of the original frequency (Kelso et al., 1998; Bourguignon et al., 2012, 2013; Nozaradan et al., 2014). These results suggest a role of slow brain oscillations in the delta frequency band $(<4 \mathrm{~Hz})$ in the control of rhythmic movements, frequencies matching the movement frequency with a 1:1 relation. Potentially conflicting results, however, come from research showing that similar modulations of slow frequency brain oscillations occur irrespective of whether participants are actively or passively producing the movement, suggesting that these modulations might result from sensory or proprioceptive feedback of the movement instead of top-down motor commands (Piitulainen et al., 2013; Bourguignon et al., 2015).

There is also evidence in the literature that brain oscillations at faster frequencies in the beta band $(\approx 20 \mathrm{~Hz})$ play an important role in the production and control of self-paced rhythmic movements. It has been demonstrated that the amplitude of beta oscillations in the primary motor cortex is dynamically modulated when performing movements, and that beta oscillations support connectivity between cortical motor areas and the peripheral muscles producing the movement (Pfurtscheller et al., 1981; Salmelin et al., 1994; Salenius et al., 1997; Hari et al., 1998; Neuper \& Pfurtscheller, 2001). Modulations of beta oscillations have also been shown when passively listening to auditory rhythms, and it has been argued that these modulations may underlie motor entrainment to auditory rhythms, and musical rhythms in particular (Fujioka et al., 2012; Merchant et al., 2015). Moreover, most of the effects of tACS reported so far on motor performances, as detailed above, have been obtained using stimulation frequencies in the beta range (Pogosyan et al., 2009; Feurra et al., 2011; Wach et al., 2013). Effects on the variability of finger tapping have been obtained using $20 \mathrm{~Hz}-$ 
frequency stimulations (Wach et al., 2013). Enhanced MEPs occurring with tACS are also frequency-dependent and have been found to occur reliably with $20 \mathrm{~Hz}$ stimulations (Feurra et al., 2011). Taken together, these results support that stimulation targeting beta oscillations might be effective at influencing the dynamics of self-paced rhythmic movements.

Across two experiments, the current study investigated the effects of tACS on selfpaced rhythmic movements when applied over the primary motor cortex (M1). Specifically, we investigated whether tACS could entrain and modify the movement dynamics of participants oscillating a handheld pendulum at their preferred movement tempo. In Experiment 1, we examined the effects of tACS with slow frequency stimulations. The stimulation was delivered at a frequency equal to participant's preferred movement frequency that was prerecorded (i.e., around $1 \mathrm{~Hz}$ ) or slightly faster (preferred frequency $+12.5 \%$ ) and slower (preferred frequency $-12.5 \%$ ) to determine whether 1:1 movement-stimulation frequency relation strengthens tACS effects. We expected that stimulation at participant's preferred frequency would result in stronger phase entrainment whereas stimulation at preferred frequency $\pm 12.5 \%$ would attract participant's movement to faster and slower tempi, respectively. In experiment 2, we examined the effects of tACS with faster stimulation frequencies in the beta $(\approx 20 \mathrm{~Hz})$ range. We also tested stimulations in the alpha $(\approx 10 \mathrm{~Hz})$ range to address the hypothesis that not any type of faster stimulation would induce movement modification but only stimulation in the beta range that is particularly related to motor processes. Stimulations were delivered at $10 \mathrm{~Hz}$ and $10 \mathrm{~Hz} \pm 12.5 \%$ (i.e., 8.75 or $11.25 \mathrm{~Hz}$ ) for alpha and at $20 \mathrm{~Hz}$ and $20 \mathrm{~Hz} \pm 12.5 \%$ (i.e., 17.5 or $22.5 \mathrm{~Hz}$ ) for beta, with the expectation that stimulation at the base frequencies, especially for beta $(20 \mathrm{~Hz})$, would result in stronger movement entrainment and stabilization (decreased frequency and amplitude variability) compared to stimulation at the lower and higher frequencies, which were expected to lead to movement attraction to faster and slower tempi, respectively.

\section{Experimental Procedures}

\section{Participants}

Twenty-four right-handed adults volunteered to participate in Experiment 1 (3 males and 21 females) and Experiment 2 (4 males and 20 females). Each individual participated only in one of the two experiments and none of them had contradictions to brain stimulation. The mean age of the participants was $22.71(S D=6.77)$ and $23.46(S D=7.04)$ in Experiment 1 and 2, respectively. They all provided written informed consent prior to the experiments, which were approved by the Western Sydney University Ethics Committee. 


\section{Apparatus}

Participants, who were tested individually, were seated in a chair with their right forearm comfortably positioned on a soft support parallel to the ground. The pendulum swung by the participant in the sagittal plane using ulnar-radial deviation of the wrist joint (see Fig. 1) was constructed from a wooden dowel measuring $45 \mathrm{~cm}$ in length and had a $75-\mathrm{g}$ weight attached to its base, resulting in a gravitational eigenfrequency of $0.9 \mathrm{~Hz}$. A $1 \times 1 \times 1.5 \mathrm{~cm}$ sensor was attached to the pendulum to record participants' oscillations with a $.01 \mathrm{~mm}$ spatial resolution at a $30 \mathrm{~Hz}$ sampling rate using a Polhemus Liberty motion tracker (Polhemus Ltd., VT, USA). A computer screen positioned in front of the participant displayed paired-images during trials for a spot-the-difference concentration task used to limit large eye and head movements that could influence participants' pendulum movements (Varlet et al., 2015).

tACS was delivered over the left or right primary motor cortex (M1) of participants via a NeuroConn DC Stimulator Plus (NeuroConn Gmbh, Ilmenau, Germany) with $5 \times 7 \mathrm{~cm}$ $\left(35 \mathrm{~cm}^{2}\right)$ electrodes housed in saline soaked sponges. The saline had a concentration of Sodium Chloride: $7.67 \mathrm{~g} / \mathrm{L}$ and Disodium Hydrogen Phosphate: $2.0 \mathrm{~g} / \mathrm{L}$. Plastic syringes were used to apply further saline to sponges throughout the experiments to keep impedance low. The left primary motor cortex of participants was stimulated in Experiment 1 and 2 by positioning one electrode at the level of $\mathrm{C} 3$ according to the 10/20 international system and the second electrode on the contralateral (right) shoulder over the trapezius muscle (Mehta et al., 2015). For an additional control condition, the right primary motor cortex of participants was also stimulated in Experiment 1 by positioning electrodes at the level of $\mathrm{C} 4$ and on the contralateral (left) shoulder. The halfway point between the nasion and inion was measured and marked on the scalp to locate the vertex $(\mathrm{Cz})$. This mark was then dissected by finding the halfway point between the two preauriculars, and then $20 \%$ of this distance was calculated to locate C3 and C4 electrode positions either side of the vertex (Da Silva et al., 2011). The electrode montages were secured to the participant and remained in place throughout all trials. Cranial electrodes were bound to the head under the chin using a two-inch wide Nexcare selfadherent wrap (3M Australia P/L). Alcohol wipes were used to prepare the skin of participant's shoulder prior to the electrode attachment with hypoallergenic medical tape.

\section{Procedure}

On arrival the participant was screened for eligibility for brain stimulation protocols via a short questionnaire and given further details about the experiment before obtaining informed consent. After being seated in the chair, the participant was asked to hold firmly the 
pendulum with the right hand and to swing it from the wrist joint (radial-ulnar abductionadduction) at her/his most confortable tempo - "the tempo you could keep the all day without fatigue". Following these instructions, the participant was given a practice period to explore her/his preferred movement tempo. In experiment 1, three stimulation-free trials of $90 \mathrm{~s}$ each were then recorded to determine the participant's preferred movement tempo (average of the three trials), which was used to set the stimulation parameters for the following experimental trials (Schmidt et al., 2007; Varlet et al., 2015, 2016).

In each trial, the participant was required to swing the pendulum at the most comfortable tempo for few seconds before the recording of the pendulum movement and the stimulation commenced and lasted for 90s. The participant was instructed to maintain her or his preferred tempo while performing a spot-the-difference visual task and tACS was delivered. For the spot-the-difference task, one pair of images was displayed for the full length of each trial and the participant was asked to count the number of differences. At the end of each trial, the participant reported to the experimenter the number of differences she or he found and the tACS parameters were then changed according to the next trial condition. A 1 min-break minimum separated two consecutive trials.

In Experiment 1, two independent variables - stimulation condition (contralateral, ipsilateral and control [without stimulation]) and stimulation frequency (preferred frequency $12.5 \%$, preferred frequency, and preferred frequency $+12.5 \%$ ) were manipulated to test the effects of low-frequency tACS and stimulation-movement frequency differences. The ipsilateral stimulation condition (stimulation of right M1, not primarily involved in controlling the moving right hand) was used in addition to control trials (i.e., without stimulation) in order to examine the region-specific nature of the hypothesized tACS effects in the contralateral condition.

Figure 1 about here

In Experiment 2, two independent variables - stimulation condition (alpha, beta and control) and stimulation frequency (low, mid, and high) were manipulated to test the effects of higher-frequency tACS. Mid-range alpha and beta stimulations were delivered at $10 \mathrm{~Hz}$ and $20 \mathrm{~Hz}$, respectively, and low-range and high-range alpha and beta frequency stimulation corresponded to these base frequencies $\pm 12.5 \%$, in line with Experiment 1 . The main 
difference between experiments is therefore that stimulation frequencies were matched to the prerecorded preferred movement frequency of individual participants in Experiment 1 but were the same for all participants in Experiment 2.

The participant completed 21 experimental trials in total in both Experiment 1 and 2, which included 3 repetitions for each of the conditions contra/-12.5\%, contra/PF (PF, preferred frequency), contra/+12.5\%, ipsi/-12.5\%, ipsi/PF, ipsi/+12.5 in Experiment 1, and alpha/low $8.75 \mathrm{~Hz}$, alpha/mid 10Hz, alpha/high $11.25 \mathrm{~Hz}$, beta/low $17.5 \mathrm{~Hz}$, beta/mid $20 \mathrm{~Hz}$, beta/high $22.5 \mathrm{~Hz}$ in Experiment 2, in addition to 3 control trials without stimulation. Each trial series was divided into two main blocks - trials 2-10 and 12-20 for contralateral and ipsilateral conditions in Experiment 1 and for alpha and beta conditions in Experiment 2 with trials 1,11 , and 21 remaining fixed as control trials without stimulation. The order of the blocks was counterbalanced across participants and the trials within each block were randomized.

Stimulation was delivered at $2 \mathrm{~mA}$ peak to peak amplitude and impedances were kept below $10 \mathrm{~K} \Omega$. The number of cycles delivered was adjusted to obtain a trial duration of $90 \mathrm{~s}$, including a ramp up/down of approximately $3 \mathrm{~s}$. The participant was kept blind to experimental conditions. None of the participants reported being aware of stimulation versus control conditions when debriefed at the end of the experiment.

After finishing the experimental trials in Experiment 1 and 2, the participant was asked to complete a tACS side effects questionnaire, adapted from Brunoni et al. (2011). The severity of items such as tingling, itching, burning, and drowsiness were recorded (Absent, Mild, Moderate, Severe) (See Table 1). The participant was then debriefed about the research questions under investigation before leaving.

\section{Data analysis}

The recorded movement time series were centered around zero and low-pass filtered using a third-order bidirectional $10 \mathrm{~Hz}$ Butterworth filter. The first $10 \mathrm{~s}$ of each trial was discarded to eliminate transient behavior (Schmidt et al., 2007; Varlet et al., 2015). The time between the points of maximum angular extension as defined by the maxima of the movement time series was then computed to determine the average preferred movement frequency of participants and the corresponding coefficient of variation $(\mathrm{COV}=$ $\mathrm{SD} / \mathrm{Mean} \times 100$ ), indexing the magnitude of the frequency variability inherent to their movement. The distance between maximum angular flexion and extension of each cycle, as 
defined by the difference between two consecutive minima and maxima of the movement time series, was used to compute the average movement amplitude and the corresponding coefficient of variation. As the movement frequency and amplitude tended to exhibit slow drifts throughout trials, frequency and amplitude COVs were computed both before and after linear detrending the frequency and amplitude time series in order to index both global and local variability, respectively.

The phase synchronization between the delivered tACS signal and participant's pendulum movement was assessed in Experiment 1 by computing the continuous relative phase between the two time series using the Hilbert Transform. Circular statistics were then used to compute the circular variance of the relative phase, giving an index of synchrony between 0 and 1, where 0 equals no synchrony and 1 equals perfect synchrony (Batschelet, 1981; Tognoli et al., 2007; Varlet et al., 2016). Movement synchronization to the stimulation signal was not directly measured in Experiment 2 due to the fast frequencies delivered, which implies more complex forms of synchrony on frequency ratios and requires more complex analyses (e.g., Washburn et al., 2014). As the frequency of the tACS signal remained fixed throughout the trials, the occurrence of entrainment on these more complex forms of synchrony was however expected to be reflected in the average and variability of participants' movement frequency. Accordingly, the dependent variables included: 1) mean frequency, 2) frequency COV, 3) mean amplitude, 4) amplitude COV and 5) phase synchrony (the latter was computed for Experiment 1 only).

These dependent variables were submitted to 2-way repeated measures ANOVAs with the factors Stimulation Condition (contralateral, ipsilateral and control) and Stimulation Frequency (preferred frequency $-12.5 \%$, preferred frequency, and preferred frequency $+12.5 \%$ ) in Experiment 1 and with the factors Stimulation Condition (alpha, beta and control) and Stimulation Frequency (low, mid, and high) in Experiment 2. The same three control trials (\# 1, 11 and 21) were used to compute each dependent variable in the different conditions. The phase synchrony computed in Experiment 1 in these control trials was obtained by combining the participant's movement recorded in these trials with the tACS signals presented in the corresponding experimental conditions. These trials served to index the level of synchrony between participant's movement and the stimulation signal that occurs incidentally by chance, which changes depending on the stimulation frequency delivered. The nature of the effects was examined using Bonferroni post-hoc comparisons when necessary 
and Chi-square tests were performed on tACS side effects questionnaire data to examine differences between low and higher frequency stimulations in Experiment 1 and 2.

\section{Results}

In line with previous research, the analysis of participants' movement frequency data in the control trials showed an average preferred frequency close to the pendulum's eigenfrequency. The mean frequency of participants in controls trials was $1.02(\mathrm{SD}=.10)$ in Experiment 1 and $1.05(\mathrm{SD}=.11)$ in Experiment 2. One participant in each experiment was identified as outlier because of much faster frequency than the others (+ $3 \mathrm{SD}$ from the mean) and removed from further analyses.

\section{Experiment 1}

Movement Frequency. The ANOVA performed on the mean frequency of participants' movements did not reveal any significant main effect of Stimulation Condition, $F(2,44)=$ $2.59, p>.05, \eta_{p}{ }^{2}=.10$, Stimulation Frequency, $\left(F(2,44)=.25, p>.05, \eta_{p}{ }^{2}=.01\right.$, or interaction between these two factors, $F(4,88)=.27, p>.05, \eta_{p}{ }^{2}=.01$. The ANOVA performed on COV Frequency did not show any significant main effects or interaction after and before detrending frequency time series - Stimulation Condition, $F(2,44)=.04, p>.05$, $\eta_{p}{ }^{2}=.002$ and $F(2,44)=.20, p>.05, \eta_{p}{ }^{2}=.01$; Stimulation Frequency, $F(2,44)=.20, p>$ $.05, \eta_{p}{ }^{2}=.01$ and $F(2,44)=1.40, p>.05, \eta_{p}{ }^{2}=.06$, or Stimulation Condition $\times$ Stimulation Frequency, $F(4,88)=2.14, p>.05, \eta_{p}{ }^{2}=.01$ and $F(4,88)=1.00, p>.05, \eta_{p}{ }^{2}=.04$, respectively.

Figure 2 about here

Movement Amplitude. The ANOVA performed on participants' mean movement amplitude data did not yield any significant main effect of Stimulation Condition, $F(2,44)=.19, p>$ $.05, \eta_{p}{ }^{2}=.01$, Stimulation Frequency, $F(2,44)=.19, p>.05, \eta_{p}^{2}=.01$, or interaction between these two factors, $F(4,88)=.25, p>.05, \eta_{p}{ }^{2}=.01$. No significant effects were revealed on movement amplitude variability at local or global levels - Stimulation Condition, $F(2,44)=2.36, p>.05, \eta_{p}{ }^{2}=.10$ and $F(2,44)=.81, p>.05, \eta_{p}{ }^{2}=.03$, Stimulation Frequency, $F(2,44)=1.29, p>.05, \eta_{p}{ }^{2}=.06$ and $F(2,44)=.77, p>.05, \eta_{p}{ }^{2}=.03$, and Stimulation Condition $\times$ Stimulation Frequency, $F(4,88)=1.00, p>.05, \eta_{p}{ }^{2}=.04$ and $F(4$, 
$88)=.29, p>.05, \eta_{p}{ }^{2}=.01$, for COVs computed on detrended and nondetrended amplitude time series, respectively.

Movement Synchrony. The ANOVA performed on the phase synchrony yielded a significant main effect of Stimulation Frequency, $F(2,44)=22.02, p<.05, \eta_{p}{ }^{2}=.50$, indicating greater synchrony in the $0 \%$ condition compared to $\pm 12.5 \%$ conditions $(p$ values $<.001)$. This increased synchrony in the $0 \%$ condition is however only the result of the frequencies of the stimulation and movement time series being close to each other in this condition. Indeed, this effect also occurred in control trials in which stimulations were actually not delivered and thus is purely incidental. The ANOVA did not show any significant main effect of Stimulation Condition, $F(2,44)=.08, p>.05, \eta_{p}{ }^{2}=.004$, or significant interaction between Stimulation Condition and Stimulation Frequency, $F(4,88)=.79, p>.05, \eta_{p}{ }^{2}=.03$, indicating that tACS had no effects on the degree of synchrony exhibited whatever the stimulation frequency.

\section{Experiment 2}

Movement Frequency. The ANOVA performed on participants' mean movement frequency data did not show any significant main effects of Stimulus Condition, $F(2,44)=1.42, p>.05$, $\eta_{p}{ }^{2}=.06$, Stimulus Frequency, $F(2,44)=.02, p>.05, \eta_{p}{ }^{2}=.001$, or significant Stimulation Condition $\times$ Stimulation Frequency interaction, $F(4,88)=.08, p>.05, \eta_{p}{ }^{2}=.004$. No significant effects were revealed by the ANOVAs performed on the COVs computed on detrended and nondetrended frequency time series - Stimulus Condition, $F(2,44)=.11, p>$ $.05, \eta_{p}{ }^{2}=.005$ and $F(2,44)=.47, p>.05, \eta_{p}{ }^{2}=.02$; Stimulus Frequency, $F(2,44)=2.73, p>$ $.05, \eta_{p}{ }^{2}=.11$ and $F(2,44)=1.20, p>.05, \eta_{p}{ }^{2}=.05 ;$ Stimulation Condition $\times$ Stimulation Frequency interaction, $F(4,88)=1.10, p>.05, \eta_{p}{ }^{2}=.05$ and $F(4,88)=1.68, p>.05, \eta_{p}{ }^{2}=$ .07 , respectively.

Figure 3 about here

Movement Amplitude. No significant effects were revealed by the ANOVA performed on participants' mean amplitude data, Stimulus Condition, $F(2,44)=2.20, p>.05, \eta_{p}{ }^{2}=.09$; Stimulus Frequency, $F(2,44)=1.28, p>.05, \eta_{p}{ }^{2}=.05$; Stimulation Condition $\times$ Stimulation Frequency, $F(4,88)=.39, p>.05, \eta_{p}{ }^{2}=.02$. The ANOVA performed on COV did not yield 
significant effects - Stimulus Condition, $F(2,44)=.06, p>.05, \eta_{p}^{2}=.003$ and $F(2,44)=.11$, $p>.05, \eta_{p}{ }^{2}=.005 ;$ Stimulus Frequency, $F(2,44)=.73, p>.05, \eta_{p}{ }^{2}=.03$ and $F(2,44)=1.32$, $p>.05, \eta_{p}{ }^{2}=.06$; Stimulation Condition $\times$ Stimulation Frequency, $F(4,88)=.41, p>.05$, $\eta_{p}{ }^{2}=.02$ and $F(4,88)=.47, p>.05, \eta_{p}{ }^{2}=.02$, for detrended and nondetrended amplitude time series, respectively.

\section{Side-effects}

The analysis of self-reported side effects (see Table 1) showed that Tingling, Itching, Drowsiness, Trouble concentrating were mentioned by participants often and with the same frequency of occurrence in Experiment 1 and 2.

Table 1 about here

Chi-square tests revealed that Burning sensations, $\chi^{2}(1, \mathrm{~N}=46)=7.22, \mathrm{p}<.05$, and Phosphenes, $\chi^{2}(1, \mathrm{~N}=46)=14.46, \mathrm{p}<.001$, were also often reported, but mostly in Experiment 2 ( 9 vs. $43 \%$ and 0 vs. $48 \%$, respectively), whereas Metallic taste, which was occasionally reported, tended to be experienced only in Experiment $1, \chi^{2}(1, N=46)=3.21, p$ $<.1$. These results suggest that some side effects induced by tACS are frequency-dependent and others are not.

\section{Discussion}

Across two experiments, the current study investigated whether tACS can be used to entrain or modulate the dynamics of self-paced rhythmic movements. While participants oscillated a handheld pendulum at their preferred movement tempo, stimulations were delivered at the level of their left or right primary motor cortex at frequencies corresponding to their actual movement frequency (Experiment 1) or at frequencies in the alpha and beta ranges (Experiment 2). Neither of the two experiments revealed evidence of entrainment or modulation of participants' movement. The mean and variability of participants' movement frequency and amplitude were not affected by tACS. Significant differences were only revealed between Experiment 1 and 2 for the side effects induced by tACS, which are generally in line with side effects previously reported (Brunoni et al., 2011; Wach et al., 2013). Interestingly, burning sensation and phosphenes were frequency-dependent and principally occurred in Experiment 2 with faster stimulation frequencies in the alpha and beta 
ranges, whereas metallic tastes were only experienced by some participants in Experiment 1 with low frequency stimulations. Together, these results provide further insights into side effects potentially induced by brain stimulation methods, transcranial current stimulation in particular, complementing existing safety and application guidelines (Nitsche et al., 2003; Rossi et al., 2009; Brunoni et al., 2011; Chaieb et al., 2014).

The absence of effects on participants' movement suggests that inducing movement entrainment or modulation directly via rhythmic direct-current brain stimulation is not as straightforward as via visual and auditory rhythmic stimulations. Further research will be necessary before being able to replace or complement visual and auditory cueing by tACS to entrain and enhance human rhythmic motor performance.

Different reasons could explain the absence of effects of tACS in the current study but it is first important to note that the absence of effects on participants' movement does not demonstrate the absence of effects of tACS on participants' brain activity. Indeed, it remains possible that tACS entrained or modulated brain activity as expected but the modulations were too weak, too variable, or not focused on processes underlying the production and control of the movements produced by participants. In particular, there is growing evidence in the motor control literature showing that the production and control of rhythmic movements involves different neurophysiological processes depending on movement properties (Capaday et al., 2002; Spencer et al., 2003; Schaal et al., 2004; Torre \& Balasubramaniam, 2009). Continuous oscillatory movements, strongly driven by external mechanical constraints, such as pendulum swinging in the current study, are known to involve less central control processes than discontinuous rhythmic movements such as finger tapping, for which tACS effects have been previously reported (Wach et al., 2013; Novembre et al., in press). Moreover, mechanical constraints in pendulum swinging tasks lead to high movement stability - more than in finger tapping tasks, for instance, in which movement flexibility is greater - leaving open the possibility that entrainment induced by tACS might have occurred if our participants had performed a less constrained movement. Although future research is required to confirm such differential effects of tACS on continuous and discontinuous movements, it may be the case that tACS, and electrical stimulation of the central nervous system more generally, might be of limited potential in modulating everyday motor behaviors such as locomotion and postural performances involving continuous oscillatory movements strongly driven by peripheral constraints. The use of electroencephalography (EEG) will be of 
particular interest in future research to measure entrainment not only at a behavioral level but also at a neural level in order to fully address these questions (Helfrich et al., 2014).

Although a range of stimulation frequencies was explored across the two experiments of the current study, the fact that some of the stimulation parameters were not manipulated could have contributed to the absence of effects. First, the stimulation intensity remained set at $2 \mathrm{~mA}$ in the two experiments. Although this intensity is in line with the 1 or $2 \mathrm{~mA}$ usually used, previous research has shown that small intensity differences can significantly change tACS effects induced (Moliadze et al., 2012). Second, the stimulation duration remained fixed at 90s, which corresponds to the stimulation duration used in the study of Feurra et al. (2011) that showed significant modulations of the size of the MEPs induced by TMS. It is also longer than the 40-60s of visual or auditory rhythmic stimulations often used to investigate sensorimotor entrainment (Schmidt et al., 1997; Richardson et al., 2007; Varlet et al., 2015, 2016). However, several effects of tACS have been found using longer stimulation durations, such as 10min (Zaghi et al., 2010; Wach et al., 2013; Helfrich et al., 2014), which encourages the use of such durations in future research testing movement entrainment. Finally, the position of the electrodes remained identical throughout the study and the particular montage used might have contributed to the absence of effects. Electrodes were positioned on the primary motor cortex of participants at the level of C3 and C4 according to the 10/20 international system and the second electrode was positioned on the contralateral shoulder over the trapezius muscle. This position of the second electrode was chosen because it was found to be most effective in modulating movements and creating the least amount of phosphenes in previous research (Mehta et al., 2015). However, several significant effects of tACS on motor output have been shown using other montages where the second electrode was also positioned on the head (Feurra et al, 2011; Wach, 2013), which suggests that such placement could similarly facilitate the occurrence of movement entrainment under conditions tested in the current study.

To conclude, this study demonstrates a lack of effects of tACS on the entrainment or modulation of human rhythmic movements, with significant effects occurring only at the level of the side effects induced by low and higher stimulation frequencies in Experiment 1 and 2, respectively. Our findings suggest that there is need for further investigation of the effects of tACS on human rhythmic movements using systematic manipulations of the type of movement produced and stimulation parameters. Such a systematic approach is necessary in 
order to better determine the potential benefits of tACS for entraining and enhancing everyday rhythmic motor behaviors and helping motor rehabilitation in clinical populations.

\section{Acknowledgement}

P.K. is supported by a Future Fellowship grant from the Australian Research Council (FT140101162). 


\section{Reference}

Antal A, Boros K, Poreisz C, Chaieb L, Terney D, Paulus, W (2008), Comparatively weak after-effects of transcranial alternating current stimulation (tACS) on cortical excitability in humans. Brain Stim 1: 97-105.

Antal A, Paulus W (2013), Transcranial alternating current stimulation (tACS). Front Hum Neurosci 7: 317.

Batschelet E (1981), Circular statistics in biology (Vol. 371). Academic Press London.

Beek PJ, Schmidt RC, Morris AW, Sim M, Turvey MT (1995), Linear and nonlinear stiffness and friction in biological rhythmic movements. Biol Cybern 73: 499-507.

Bourguignon M, De Tiège X, Op de Beeck M, Van Bogaert P, Goldman S, Jousmäki V, Hari R (2013), Primary motor cortex and cerebellum are coupled with the kinematics of observed hand movements. NeuroImage 66: 500-507.

Bourguignon M, Jousmäki V, de Beeck MO, Van Bogaert P, Goldman S, De Tiège X (2012), Neuronal network coherent with hand kinematics during fast repetitive hand movements. NeuroImage 59: 1684-1691.

Bourguignon M, Piitulainen H, De Tiège X, Jousmäki V, Hari R (2015), Corticokinematic coherence mainly reflects movement-induced proprioceptive feedback. NeuroImage 106: $382-390$.

Brittain JS, Probert-Smith P, Aziz TZ, Brown P (2013), Tremor suppression by rhythmic transcranial current stimulation. Cur Biol 23: 436-440.

Brunoni AR, Amadera J, Berbel B, Volz MS, Rizzerio BG, Fregni F (2011), A systematic review on reporting and assessment of adverse effects associated with transcranial direct current stimulation. Int J Neuropsychopharm 14: 1133-1145.

Capaday C (2002), The special nature of human walking and its neural control. Trends Neurosci 25: 370-376.

Chaieb L, Antal A, Pisoni A, Saiote C, Opitz A, Ambrus GG, Focke N, Paulus W (2014), Safety of 5 kHz tACS. Brain Stim 7: 92-96.

Cheyne D, Bells S, Ferrari P, Gaetz W, Bostan AC (2008), Self-paced movements induce high-frequency gamma oscillations in primary motor cortex. NeuroImage 42: 332342. 
Coey C, Varlet M, Schmidt RC, Richardson MJ (2011), Effects of movement stability and congruency on the emergence of spontaneous interpersonal coordination. Exp Brain Res 211: 483-493.

Da Silva AF, Volz MS, Bikson M, Fregni F (2011), Electrode positioning and montage in transcranial direct current stimulation. JoVE 51: e2744.

Feurra M, Bianco G, Santarnecchi E, Del Testa M, Rossi A, Rossi S (2011), Frequencydependent tuning of the human motor system induced by transcranial oscillatory potentials. J Neurosci 31: 12165-12170.

Fröhlich F (2014), Endogenous and exogenous electric fields as modifiers of brain activity: rational design of noninvasive brain stimulation with transcranial alternating current stimulation. Dialogues Clin Neurosci 16: 93.

Fujioka T, Trainor LJ, Large EW, Ross B (2012), Internalized timing of isochronous sounds is represented in neuromagnetic beta oscillations. J Neurosci 32: 1791-1802.

Guerra A, Pogosyan A, Nowak M, Tan H, Ferreri F, Di Lazzaro V, Brown P (2016), Phase Dependency of the Human Primary Motor Cortex and Cholinergic Inhibition Cancelation During Beta tACS. Cereb Cortex 26: 3977-3990.

Hari R, Forss N, Avikainen S, Kirveskari E, Salenius S, Rizzolatti G (1998), Activation of human primary motor cortex during action observation: a neuromagnetic study. Proc Natl Acad Sci USA 95 15061-15065.

Helfrich RF, Schneider TR, Rach S, Trautmann-Lengsfeld SA, Engel AK, Herrmann CS (2014), Entrainment of brain oscillations by transcranial alternating current stimulation. Cur Biol 24: 333-339.

Herrmann CS, Rach S, Neuling T, Strüber D (2013), Transcranial alternating current stimulation: a review of the underlying mechanisms and modulation of cognitive processes. Front Hum Neurosci 7: 229.

Hove MJ, Keller PE (2015), Impaired movement timing in neurological disorders: rehabilitation and treatment strategies. Ann N Y Acad Sci 1337: 111-117.

Joundi RA, Jenkinson N, Brittain JS, Aziz TZ, Brown P (2012), Driving oscillatory activity in the human cortex enhances motor performance. Cur Biol 22: 403-407. 
Keil J, Timm J, SanMiguel I, Schulz H, Obleser J, Schönwiesner M (2014), Cortical brain states and corticospinal synchronization influence TMS-evoked motor potentials. J Neurophysiol 111: 513-519.

Kelso JAS (1984), Phase transitions and critical behavior in human bimanual coordination. Am J Physiol Regul Integr Comp Physiol 246: R1000-R1004.

Kelso JAS (1995), Dynamic Patterns: The Self Organization of Brain and Behaviour. The MIT Press.

Kelso JAS, Fuchs A, Lancaster R, Holroyd T, Cheyne D, Weinberg H (1998), Dynamic cortical activity in the human brain reveals motor equivalence. Nature 392: 814-818.

Kelso JAS, Southard DL, Goodman D (1979), On the nature of human interlimb coordination. Science 203: 1029-1031.

Krause V, Wach C, Südmeyer M, Ferrea S, Schnitzler A, Pollok B (2013), Cortico-muscular coupling and motor performance are modulated by $20 \mathrm{~Hz}$ transcranial alternating current stimulation (tACS) in Parkinson's disease. Front Hum Neurosci 7: 511.

Kugler PN, Turvey MT (1987), Information, natural law, and the self-assembly of rhythmic movement. Routledge.

Lopresti-Goodman SM, Richardson MJ, Silva PL, Schmidt RC (2008), Period basin of entrainment for unintentional visual coordination. J Motor Behav 40: 3-10.

McIntosh GC, Brown SH, Rice RR, Thaut MH (1997), Rhythmic auditory-motor facilitation of gait patterns in patients with Parkinson's disease. J Neurol Neurosurg Psychiatry 62: 22-26.

Mehta AR, Pogosyan A, Brown P, Brittain J-S (2015), Montage matters: The influence of transcranial alternating current stimulation on human physiological tremor. Brain Stim 8: $260-268$.

Merchant H, Grahn J, Trainor L, Rohrmeier M, Fitch WT (2015), Finding the beat: a neural perspective across humans and non-human primates. Phil Trans R Soc B 370: 20140093.

Moliadze V, Atalay D, Antal A, Paulus W (2012), Close to threshold transcranial electrical stimulation preferentially activates inhibitory networks before switching to excitation with higher intensities. Brain Stim 5: 505-511. 
Nakazono H, Ogata K, Kuroda T, Tobimatsu S (2016), Phase and Frequency-Dependent Effects of Transcranial Alternating Current Stimulation on Motor Cortical Excitability. PloS One 11: e0162521.

Néda Z, Ravasz E, Brechet Y, Vicsek T, Barabási A-L (2000), Self-organizing processes: The sound of many hands clapping. Nature 403: 849-850.

Nessler JA, Gilliland SJ (2009), Interpersonal synchronization during side by side treadmill walking is influenced by leg length differential and altered sensory feedback. Hum Mov Sci 28: 772-785.

Neuper C, Pfurtscheller G (2001), Evidence for distinct beta resonance frequencies in human EEG related to specific sensorimotor cortical areas. Clin Neurophysiol 112: 20842097.

Nitsche MA, Liebetanz D, Lang N, Antal A, Tergau F, Paulus W (2003), Safety criteria for transcranial direct current stimulation (tDCS) in humans. Clin Neurophysiol 114: 2220-2222.

Nombela C, Hughes LE, Owen AM, Grahn JA (2013), Into the groove: can rhythm influence Parkinson's disease?. Neurosci Biobehav Rev 37: 2564-2570.

Novembre G, Knoblich G, Dunne L, Keller P (in press), Interpersonal synchrony enhanced through $20 \mathrm{~Hz}$ phase-coupled dual brain stimulation. Soc Cogn Affect Neurosci.

Nozaradan S (2014), Exploring how musical rhythm entrains brain activity with electroencephalogram frequency-tagging. Philos Trans R Soc of London B Biol Sci 369: 20130393.

Pfurtscheller G (1981), Central beta rhythm during sensorimotor activities in man. Electroencephalogr Clin Neurophysiol 51: 253-264.

Piitulainen H, Bourguignon M, De Tiège X, Hari R, Jousmäki V (2013), Corticokinematic coherence during active and passive finger movements. Neuroscience 238: 361-370.

Pikovsky A, Rosenblum M, Kurths J (2003), Synchronization: a universal concept in nonlinear sciences (Vol. 12). Cambridge university press.

Pogosyan A, Gaynor LD, Eusebio A, Brown P (2009), Boosting cortical activity at beta-band frequencies slows movement in humans. Cur Biol 19: 1637-1641. 
Pogosyan A, Yoshida F, Chen CC, Martinez-Torres I, Foltynie T, Limousin P, Zrinzo L, Hariz, MI, Brown P (2010), Parkinsonian impairment correlates with spatially extensive subthalamic oscillatory synchronization. Neuroscience 171: 245-257.

Pollok B, Boysen AC, Krause V (2015), The effect of transcranial alternating current stimulation (tACS) at alpha and beta frequency on motor learning. Behav Brain Res 293: 234-240.

Raco V, Bauer R, Tharsan S, Gharabaghi A (2016), Combining TMS and tACS for ClosedLoop Phase-Dependent Modulation of Corticospinal Excitability: A Feasibility Study. Front Cell Neurosci 10: 1-8.

Reato D, Rahman A, Bikson M, Parra LC (2013), Effects of weak transcranial alternating current stimulation on brain activity — a review of known mechanisms from animal studies. Front Hum Neurosci 7: 687.

Repp BH, Penel A (2004), Rhythmic movement is attracted more strongly to auditory than to visual rhythms. Psychol Res 68: 252-270.

Richardson MJ, Marsh KL, Isenhower RW, Goodman JR, Schmidt RC (2007), Rocking together: Dynamics of intentional and unintentional interpersonal coordination. Hum Mov Sci 26: 867-891.

Rossi S, Hallett M, Rossini PM, Pascual-Leone A, Safety of TMS Consensus Group (2009), Safety, ethical considerations, and application guidelines for the use of transcranial magnetic stimulation in clinical practice and research. Clin Neurophysiol 120: 20082039.

Salenius S, Portin K, Kajola M, Salmelin R, Hari R (1997), Cortical control of human motoneuron firing during isometric contraction. J Neurophysiol 77: 3401-3405.

Salmelin R, Hari R (1994), Spatiotemporal characteristics of sensorimotor neuromagnetic rhythms related to thumb movement. Neuroscience 60: 537-550.

Schaal S, Sternad D, Osu R, Kawato M (2004), Rhythmic arm movement is not discrete. Nature Neurosci 7: 1136-1143.

Schmidt RC, Bienvenu M, Fitzpatrick PA, Amazeen PG (1998), A comparison of intra-and interpersonal interlimb coordination: Coordination breakdowns and coupling strength. J Exp Psychol: HPP 24: 884-900. 
Schmidt RC, O’Brien B (1997), Evaluating the dynamics of unintended interpersonal coordination. Ecol Psychol 9: 189-206.

Schmidt RC, Richardson MJ (2008), Dynamics of interpersonal coordination. In Coordination: Neural, behavioral and social dynamics (Fuchs A, Jirsa VK, eds), pp 281-308, Springer.

Schmidt RC, Richardson MJ, Arsenault C, Galantucci B (2007), Visual tracking and entrainment to an environmental rhythm. J Exp Psychol: HPP 33: 860-870.

Spencer RM, Zelaznik HN, Diedrichsen J, Ivry RB (2003), Disrupted timing of discontinuous but not continuous movements by cerebellar lesions. Science 300: 1437-1439.

Thaut MH, McIntosh GC, Rice RR, Miller RA, Rathbun J, Brault JM (1996), Rhythmic auditory stimulation in gait training for Parkinson's disease patients. Mov Disorders 11: 193-200.

Torre K, Balasubramaniam R (2009), Two different processes for sensorimotor synchronization in continuous and discontinuous rhythmic movements. Exp Brain Res 199: 157-166.

Tognoli E, Lagarde J, DeGuzman GC, Kelso JS (2007), The phi complex as a neuromarker of human social coordination. Proc Natl Acad Sci USA 104: 8190-8195.

Varlet M, Bucci C, Richardson MJ, Schmidt RC (2015), Informational constraints on spontaneous visuomotor entrainment. Hum Mov Science 41: 265-281.

Varlet M, Richardson MJ (2015), What would be Usain Bolt's 100-meter sprint world record without Tyson Gay? Unintentional interpersonal synchronization between the two sprinters. J Exp Psychol: HPP 41: 36-41.

Varlet M, Schmidt RC, Richardson MJ (2016), Influence of internal and external noise on spontaneous visuomotor synchronization. J Motor Behav 48: 122-141.

Varlet M, Stoffregen TA, Chen FC, Alcantara C, Marin L, Bardy BG (2014), Just the sight of you: Postural effects of interpersonal visual contact at sea. J Exp Psychol: HPP 40: 2310-2318.

von Holst E (1973), Relative coordination as a phenomenon and as a method of analysis of central nervous system function. In: The collected papers of Erich von Holst: Vol. 1. 
The behavioral physiology of animal and man (Martin R, ed and trans), pp 33-135, Coral Gables, FL: University of Miami Press. (Original work published 1939)

Wach C, Krause V, Moliadze V, Paulus W, Schnitzler A, Pollok B (2013), Effects of 10Hz and $20 \mathrm{~Hz}$ transcranial alternating current stimulation (tACS) on motor functions and motor cortical excitability. Behav Brain Res 241: 1-6.

Washburn A, Coey CA, Romero V, Richardson MJ (2014), Visual multifrequency entrainment: can 1: 2, 2: 3, and 3: 4 coordination occur spontaneously?. J Motor Behav 46: 247-257.

Zaghi S, de Freitas Rezende L, Oliveira LM, El-Nazer R, Menning S, Tadini L, Fregni F (2010), Inhibition of motor cortex excitability with $15 \mathrm{~Hz}$ transcranial alternating current stimulation (tACS). Neurosci Let 479: 211-214. 


\section{Figure captions}

Fig 1. Illustrations of the experimental setups and designs of Experiment 1 and 2. tACS was delivered over participant's primary motor cortex (M1) when swinging a handheld pendulum with the right hand (C). Left or right M1 was stimulated in Experiment 1 (A) at frequency matching participant's preferred movement tempo, slightly faster $(+12.5 \%)$ or slower ($12.5 \%)$, while in Experiment 2 (B) left M1 only was stimulated at alpha $(10 \mathrm{~Hz}$ and $10 \mathrm{~Hz} \pm$ $12.5 \%)$ or beta $(20 \mathrm{~Hz}$ and $20 \mathrm{~Hz} \pm 12.5 \%)$ frequencies.

Fig 2. Mean and coefficient of variation of participants' movement frequency (A and B) and amplitude (C and D), and synchrony with the tACS signal (E), as a function of the different stimulation and frequency conditions in Experiment 1. The COVs represented are those computed on detrended frequency and amplitude time series, indexing local variability. The error bars represent the standard error of the mean.

Fig 3. Mean and coefficient of variation of participants' movement frequency (A and B) and amplitude (C and D) as a function of the different stimulation and frequency conditions in Experiment 2. The COVs represented are those computed on detrended frequency and amplitude time series, indexing local variability. The error bars represent the standard error of the mean. 
Figure 1

A Contra- \& ipsilateral tACS
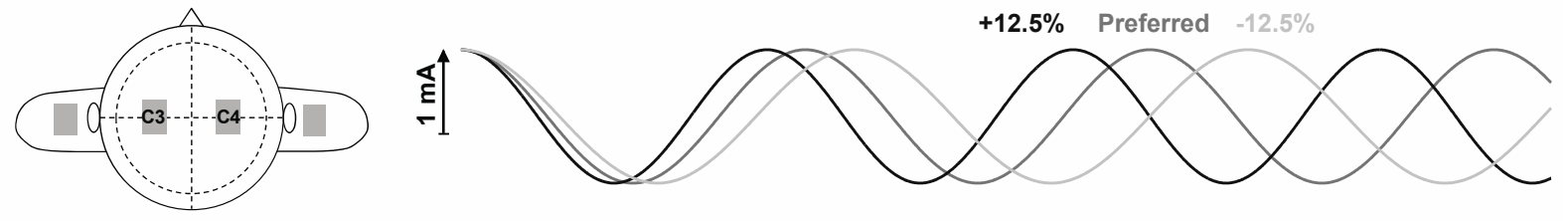

B Contralateral tACS

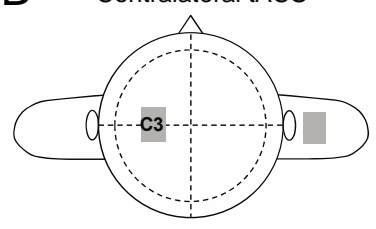

Alpha

Beta

(17.25Hz

C
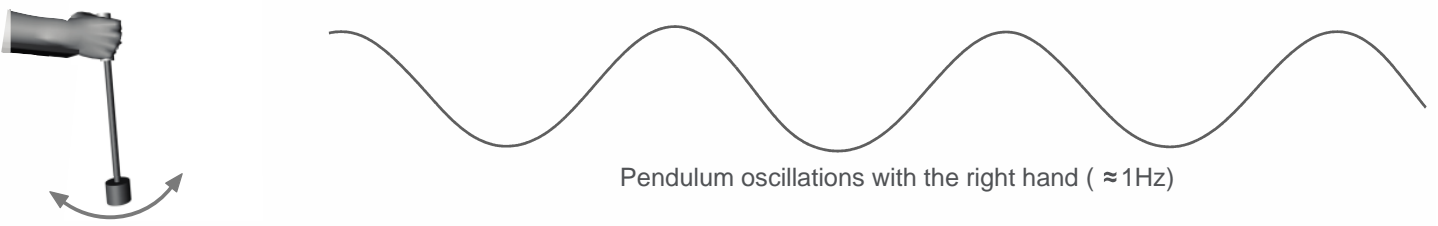
Figure 2
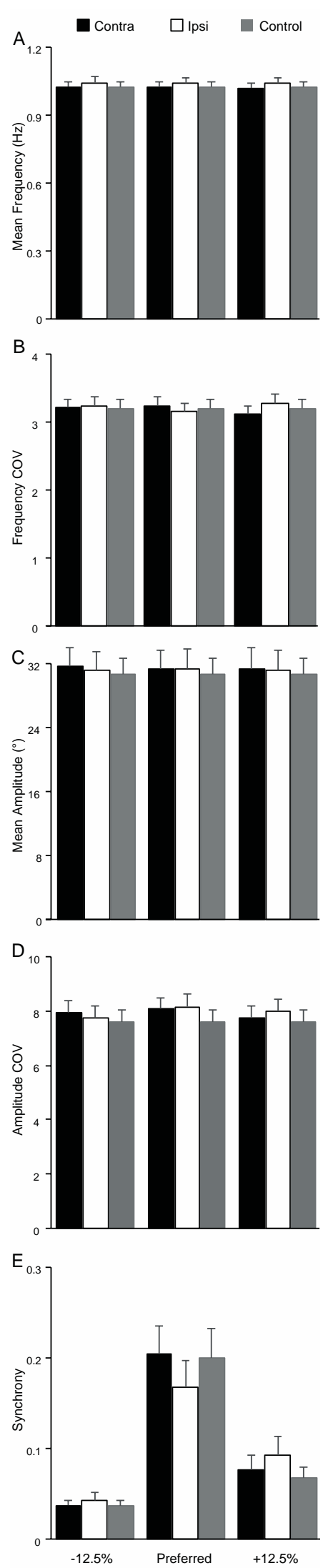
Figure 3
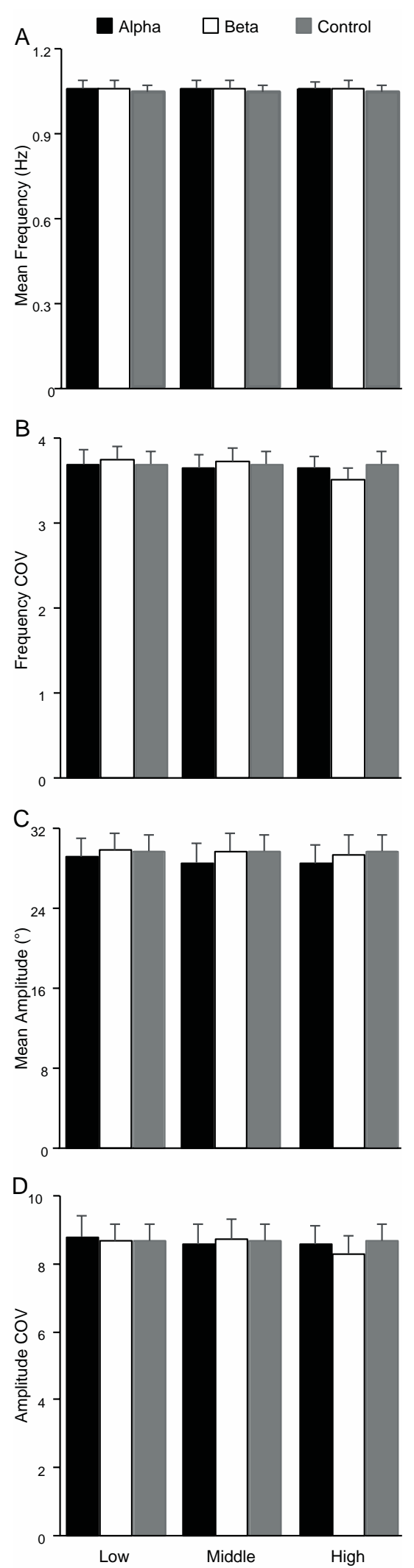


\section{Table 1}

Table 1. Side effects reported by participants in Experiment 1 and 2.

\begin{tabular}{lcccc}
\hline \multirow{2}{*}{ Side effects } & Mild & Moderate & Severe & Total \\
\cline { 2 - 5 } & Exp. $1 / 2$ & Exp. $1 / 2$ & Exp. $1 / 2$ & Exp. $1 / 2$ \\
\hline Tingling sensation & $8 / 5$ & $8 / 9$ & $1 / 3$ & $17 / 17$ \\
\hline Burning sensation & $2 / 5$ & $0 / 3$ & $0 / 2$ & $2 / 10^{* *}$ \\
\hline Itching & $8 / 2$ & $2 / 10$ & $0 / 3$ & $10 / 15$ \\
\hline Skin redness & $2 / 2$ & $0 / 1$ & $0 / 0$ & $2 / 3$ \\
\hline Headache & $2 / 4$ & $1 / 1$ & $0 / 0$ & $3 / 5$ \\
\hline Shoulder pain & $1 / 4$ & $2 / 0$ & $1 / 0$ & $4 / 4$ \\
\hline Mood change & $1 / 2$ & $1 / 1$ & $1 / 0$ & $3 / 3$ \\
\hline Drowsiness & $4 / 5$ & $2 / 6$ & $4 / 0$ & $10 / 11$ \\
\hline Trouble concentrating & $7 / 10$ & $1 / 2$ & $2 / 0$ & $10 / 12$ \\
\hline Metallic taste & $1 / 0$ & $1 / 0$ & $1 / 0$ & $3 / 0^{*}$ \\
\hline Sweet Taste & $0 / 0$ & $1 / 0$ & $0 / 0$ & $1 / 0$ \\
\hline Prick Sensation & $1 / 0$ & $0 / 0$ & $0 / 0$ & $1 / 0$ \\
\hline Phosphenes & $0 / 6$ & $0 / 4$ & $0 / 1$ & $0 / 11^{* * *}$ \\
\hline
\end{tabular}

$*, * *$ and $* * *$ indicate significant results of Chi-square tests at $p<.1, p<.05$ and $p<.001$, respectively. $\mathrm{N}=23$ in Experiment 1 and 2. 